\title{
A feasibility study for the integration of 3D accelerometry in fall risk assessment
}

\author{
C. Soaz ${ }^{1,2}$, M. Daumer ${ }^{1,2,3}$ for the VPHOP Consortium \\ ${ }^{1}$ SLCMSR-The Human Motion Institute, Munich, Germany, soaz@slcmsr.org, daumer@slcmsr.org \\ ${ }^{2}$ Department of Electrical Engineering and Information Technology, TUM, Munich, Germany \\ ${ }^{3}$ Trium Analysis Online, Munich, Germany
}

\begin{abstract}
Current research has shown that miniaturized body worn acceleration sensors can produce comparable results to existing validated clinical gait and balance scales but they are still used mainly in a research setting. The current study shows that it is technically and logistically possible to introduce standardized accelerometry into the clinical practice in the context of an European multi-center setting. The results obtained from the acceleration signals confirm the expectations and provide more information compared to the traditional methods used to assess postural stability. Finally, the correlation between gait speed and age adds to the construct validity of our algorithms. We conclude that the actibelt ${ }^{\circledR}$ platform is a promising technology to be further developed towards a validated personalized fall risk assessment tool set.
\end{abstract}

\section{Introduction}

Falls are common among the elderly, many of them resulting in fracture or other serious injury. In addition to the high medical costs of fall-related injuries, consequences of falls include long-term impaired function and mobility, fear of falling and even premature mortality [1].

There is now a growing focus on fall risk assessment and preventive interventions. Guidelines for the prevention of falls in older people recommend that interventions should be targeted at high-risk groups [2-3]. However, how to better screen and identify individuals at high-risk of falling and evaluate the effectiveness of fall prevention programs is still a topic of discussion.

Impaired balance and poor gait ability are well-established falls risk factors. Many clinical settings, as outpatient units and rehabilitation hospitals, have limited resources and time constraints, therefore inexpensive quickly administered functional performance scales such as Timed Up and Go (TUG), Romberg's Test and Functional Reach Test are commonly used to screen for gait and balance impairment. Although some of the parameters measured with these scales are usually quantifiable (time, distance, number of steps) the degree of precision and accuracy of the results can be affected by the reaction time of the operator and the resolution of the measurement tools. In other cases, there is a large undifferentiated mid-range score in which most individuals are distributed, and the risk of falling must be subjectively judged by the clinician [4].

Current research has shown that miniaturized, low-cost, wearable acceleration sensors can produce comparable results to existing validated clinical gait and balance scales [5-7] but they are still used mainly in a research setting. Aim of this exploratory study was to show the feasibility of introducing 3D accelerometer technology in a multicenter European clinical setting and examine the potential of the tool to predict risk of future falling in elderly individuals.

\section{Methods}

\subsection{Data and Subjects}

2464 rapid clinical functional tests (Table 1) performed by 224 elderly women with osteoporosis (mean age $68.3+/-$ 7.7 years old) were collected in 4 European centers in France, Germany, Italy and Switzerland using a high precision 3D accelerometer [8-10] developed at the Sylvia Lawry Center for Multiple Sclerosis Research (SLCMSR), Munich. The data collection started in July 2010 and the SLCMSR database was internally freeze for exploratory analysis in December 2011; however, collection and transfer of new actibelt ${ }^{\circledR}$ data continued in parallel. All files went through a quality control by expert readers; 2013 tests were processed and gait and balance parameters were extracted automatically; the remaining 451 test were excluded from the analysis because some of the tests were missing in the sequence or they were not sorted as specified on the acquisition protocol by the time when they were measured. These tests are though available to be included in a follow-up analysis provided they are sorted properly or/and the code which contain the functions for the automatic analysis is modified. Finally, 110 of the 2013 tests automatically processed were not included in this exploratory analysis as the clinical data and medical history of the patients was incomplete/not available at the time of data freezing. In total, 1903 tests were used for the analysis.

\subsection{Instrumentation and Test Protocol}

In our study we used a custom-built 3D accelerometer with a sample frequency of $100 \mathrm{~Hz}$, the actibelt ${ }^{\circledR}$ [8-10]. The device is integrated in a belt buckle and placed near the center of mass of the human body (Fig. 1). 


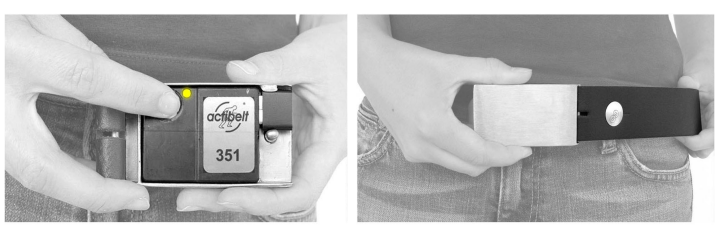

Figure 1. Photos of the actibelt ${ }^{\circledR}$ accelerometer used for the data acquisition

\subsection{Parameter Extraction of Rapid Clini- cal Tests}

All the data from the rapid test was pre-processed by noise and gravity filtering using a pass-band Butterworth filter (n $=4$, fmin $=1 / 6 \mathrm{~Hz}$, fmax $=5 \mathrm{~Hz}$ for test 1-6 and fmax $=$ $20 \mathrm{~Hz}$ for test 7-11). The parameters extracted from the actibelt ${ }^{\circledast}$ signal are described in Table 1 . For the balance tests (1-7), the analysis output includes a acceleration stabilogram which is the bidimensional plot of the left-right

\begin{tabular}{|l|l|l|}
\hline $\mathbf{N}^{\mathbf{o}}$ & Test & $\begin{array}{l}\text { Extracted actibelt } \\
\text { Number of steps, asymmetry index (3 axis), mean speed, duration, step } \\
\text { length, cadence }\end{array}$ \\
\hline 1,2 & 10 meter walk & $\begin{array}{l}\text { Number of steps, asymmetry index (3 axis), mean speed, duration, step } \\
\text { length, cadence }\end{array}$ \\
\hline 3 & $\begin{array}{l}\text { Tandem walk with cognitive task } \\
\text { (TWT) }\end{array}$ & $\begin{array}{l}\text { (\% of samples over a critical threshold) } \\
\text { duration }\end{array}$ \\
\hline 5 & TUG - self selected speed & duration \\
\hline 6 & Chair rise test & acceleration stabilogram \\
\hline 7 & Romberg stance (quiet-standing) & acceleration stabilogram \\
\hline 8 & Semi-Tandem stance & acceleration stabilogram \\
\hline 9 & Tandem stance & acceleration stabilogram \\
\hline 10 & One-legged stance & acceleration stabilogram \\
\hline 11 & One-legged stance & \\
\hline
\end{tabular}

Table 1. VPHOP list of clinical tests included in the actibelt $^{\circledR}$ clinical stability testing protocol $^{2}$

We developed the actibelt ${ }^{\circledR}$ clinical stability testing proto$\mathrm{col}$ to allow the automatic extraction of gait/balance parameters from the acceleration signal recorded with the actibelt ${ }^{\circledR}$. The ptotocol was elaborated in the context of a Research European project, The Osteoporotic Virtual Physiological Human - VPHOP. It consists in a continuous series of 11 functional clinical tests selected by an interdisciplinary expert panel to assess functional mobility in osteoporotic elderly females with focus in fall risk assessment (See Table 1) and a set of data acquisition guidelines. Beginning and end of each single test is recognized along the sequence by tapping once and twice on the sensor respectively. The DMW algorithm [11] was used to detect the peaks caused in the signal by the tapping.

Two training sessions were organized to review the protocol and instruct the practitioners. Sessions were video recorded and made available to all project partners.

The actibelt ${ }^{\circledast}$ files were uploaded by the clinicians into a web-platform for safe storage together with clinical data (demographic and anthropometric data, fracture history, retrospective fall monitoring, bone phenotype, physical activity questionnaires, musculoskeletal pain, etc).

Additionally, 7-days continuous actibelt ${ }^{\circledR}$ measurements were collected to explore possible relationships with the physical activity levels of the patients and find whether some of the parameters extracted from the rapid tests performed in clinical settings are correlated to the same ones under real life conditions. and forward-backward acceleration axis together with the ellipse that contains the $95 \%$ of the samples calculated by means of a Principal Component Analysis (PCA) transformation. The ellipse is defined by the eccentricity, the angle between the major axis and the $x$ axis and the area ( Fig. 2).

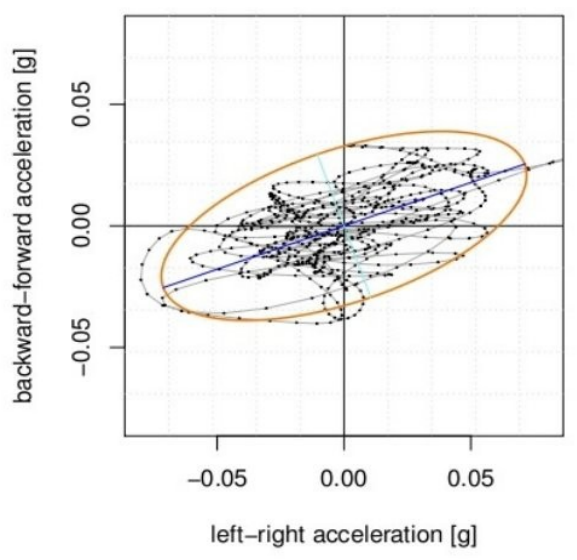

Figure 2. Acceleration stabilogram captured with the actibelt ${ }^{\circledR}$ while performing the one-legged test. 


\begin{tabular}{|l|l|l|l|l|l|}
\hline Parameter & Test & \multicolumn{5}{|c|}{ Mean value +/ - SD } & p-value \\
\hline & & Cohort1 & Cohort2 & Cohort3 \\
\hline Number of steps & 1 & $16.0+/-1.9$ & $16.5+/-1.7$ & $18.0+/-3.0$ & 0.0011 \\
\hline & 2 & $15.8+/-1.6$ & $16.4+/-1.7$ & $17.8+/-2.2$ & 0.0001 \\
\hline & 3 & $17.5+/-2.4$ & $17.5+/-2.2$ & $19.3+/-3.9$ & 0.0064 \\
\hline Speed [m/s] & 1 & $1.22+/-0.20$ & $1.19+/-0.17$ & $1.04+/-0.23$ & 0.0005 \\
\hline & 2 & $1.25+/-0.17$ & $1.22+/-0.18$ & $1.09+/-0.22$ & 0.0015 \\
\hline Step length [m] & 1 & $1.03+/-0.18$ & $1.02+/-0.24$ & $0.88+/-0.24$ & 0.0016 \\
\hline & 2 & $0.64+/-0.07$ & $0.61+/-0.06$ & $0.58+/-0.07$ & 0.0009 \\
\hline & 3 & $0.58+/-0.08$ & $0.58+/-0.07$ & $0.57+/-0.07$ & 0.0001 \\
\hline BC vert. [\%] & 4 & $1.35+/-0.40$ & $1.32+/-0.45$ & $1.58+/-0.56$ & 0.0010 \\
\hline BC lat. [\%] & 4 & $1.00+/-0.38$ & $0.99+/-0.45$ & $1.28+/-0.55$ & 0.0178 \\
\hline Duration [s] & 5 & $10.05+/-1.56$ & $10.75+/-2.14$ & $12.47+/-4.25$ & 0.0118 \\
\hline $\begin{array}{l}\text { Area [g } / 100] \\
\text { [g=m/s] }\end{array}$ & 7 & $0.04+/-0.03$ & $0.04+/-0.03$ & $0.06+/-0.04$ & 0.0127 \\
\hline & 8 & $0.06+/-0.04$ & $0.07+/-0.06$ & $0.16+/-0.24$ & 0.0000 \\
\hline & 9 & $0.17+/-0.25$ & $0.22+/-0.26$ & $0.44+/-0.47$ & 0.0000 \\
\hline & 10 & $0.34+/-0.48$ & $0.55+/-1.30$ & $0.71+/-0.87$ & 0.0001 \\
\hline
\end{tabular}

Table 3. Relationship between cohorts and actibelt ${ }^{\circledR}$ parameters

\section{Results}

Patients (173 female; mean age 69.4 +/- 4.6 years old) were divided in three age cohorts (Table 2).

\begin{tabular}{|l|l|l|}
\hline \#Cohort & Criteria & Sample size \\
\hline 1 & $60<=$ age $<65$ yrs & 41 \\
\hline 3 & $65<=$ age $<=71$ yrs & 90 \\
\hline 4 & $71<$ age $<=80$ yrs & 42 \\
\hline
\end{tabular}

Table 2. Patient cohorts

Kruskal-Wallis rank sum non-parametric tests were used to assess the association between demographic data and accelerometry parameters. Age was significantly associated to the number of steps, speed and step length for test 1 , 2 and 3. For test 4 (TWT) the BalanceCount (BC) across the vertical and lateral axis was higher for cohort 3 compared to cohort 1 and 2, as well as the test duration in the TUG test. All balance test are significantly associated with age, being the area of the acceleration stabilogram ellipse bigger as the age of the patient increases. See Table 3 .

The deviation between subjects in balance test $8-11$ is large, indicating that individuals within the groups may vary considerably across a "stability spectrum" when the difficulty of the test grows. It was also found that the asymmetry index tends to increase in the oldest group compared to the other ones.

\section{Conclusion}

The current study shows that it is technically and logistically possible to introduce standardized accelerometry into the clinical practice in the context of an European multicenter setting. The correlation between gait speed and age adds to the construct validity of our algorithms [9] and the results obtained with the acceleration stabilogram confirm the expectations and provide more information compared to the traditional methods used to assess postural stability. The outcome obtained with the traditional methods are commonly based on a "yes/no" score depending on the patient's ability to maintain balance over a time threshold examples of this are the usual outcomes for clinical balance tests as the Romberg, Tandem/Semi-Tandem or Onelegged test. That is the reason why these screening standards mostly identify the high end of the falls risk spectrum making it difficult to determine specific interventions for people which distribute along the mid-range.

The high user acceptance to wear the sensor for prolonged periods of time was remarkably high. Approximately more than $95 \%$ of patients $(n=80)$ wore the actibelt $\AA$ during the day for a continuous time of more than one week. Furthermore, it is often questioned whether assessments performed in the clinical setting are truly representative of how a given clinical intervention affects the real life of patients [12]. Therefore, the comparison of clinical and longterm datasets will be of relevant interest.

From a research perspective, we have generated a highly valuable information on gait and postural control on older adults. It is expected that these data will improve our understanding about falls risk determinants, and provide the 
supporting evidence to underpin the accuracy of falls-risk estimation. Relevant findings could be applied to improve the standardized processes and decision-support systems for falls risk management [4].

Future work of our research focuses on the refinement and validation of algorithms that allow the automatic extraction of parameters that provide an added-value compared to the usual scores - see [13] - as well as the development of an integrated accelerometry platform including webbased services and smart phone technology [14] for fall risk assessment and fall prevention to be deployed in a variety of clinical and non-clinical settings, including home.

\section{References}

[1] Swift C. Falls in late life and their consequencesimplementing effective services. BMJ. 2001;322:855857.

[2] Feder G, Cryer C, Donovan S, Carter Y on behalf of the Guidelines' Development Group. Guidelines for the prevention of falls in people over 65 . Br Med J 2000; 321: 1007-1011.

[3] American Geriatrics Society, British Geriatrics Society and American Academy of Orthopaedic Surgeons Panel on Falls Prevention. Guideline for the prevention of falls in older persons. J Am Geriatr Soc 2001; 49: 664-672.

[4] Ni Scanaill C, Garattini C, Greene BR, McGrath MJ. Technology Innovation Enabling Falls Risk Assessment in a Community Setting. Ageing Int. 2011; 36(2): 217-231. DOI: 10.1007/s12126-0109087-7 PMID: 21660088

[5]Giansanti, D. (2006). Investigation of fall-risk using a wearable device with accelerometers and rate gyroscopes. Physiological Measurement, 27, 10811090.

[6 ]Narayanan, M. R., Redmond, S. J., Scalzi, M. E., Lord, S. R., Celler, B. G., \& Lovell Ast, N. H. (2010). Longitudinal falls-risk estimation using triaxial accelerometry. IEEE Transactions on Biomedical Engineering, 57(3), 534-541.

[7] O’Sullivan, M., Blake, C., Cunningham, C., Boyle, G., \& Finucane, C. (2009). Correlation of accelerometry with clinical balance tests in older fallers and non-fallers. Age and Ageing, 38, 308-313.

[8] Daumer M, Thaler K, Kruis E, Feneberg W, Staude G, Scholz M. Steps towards a miniaturized, robust and autonomous measurement device for the long-term monitoring of the activity of patients - ActiBelt. Biomedizinische Technik/Biomedical Engineering. 2007; 52: 149-155

[9] Schimpl M, Lederer C, Daumer M, 2011 Development and Validation of a New Method to Measure Walking Speed in Free-Living Environments Using the Actibelt ${ }^{\circledR}$ Platform. PLoS ONE 6(8): e23080. doi:10.1371/journal.pone.0023080
[10]Schimpl M, Moore C, Lederer C, Neuhaus A, Sambrook J, et al. 2011 Association between Walking Speed and Age in Healthy, Free-Living Individuals Using Mobile Accelerometry-A Cross-Sectional Study. PLoS ONE 6(8): e23299. doi:10.1371/journal.pone.0023299

[11]Daumer, M. and Neiss, A. A new adaptive algorithm to detect shifts, drifts and outliers in biomedical time series. Mathematical Statistics with Applications in Biometry, Josef Eul, Lohmar, 2001. pp. 201-204.

[12]Bonato, Advances in wearable technology and applications in physical medicine and rehabilitation, Journal of NeuroEngineering and Rehabilitation 2 (1) (2005), 2.

[13]Weiss A, Herman T, Plotnik M, Brozgol M, Giladi N, Hausdorff .An instrumented timed up and go: the added value of an accelerometer for identifying fall risk in idiopathic fallers. JM.Physiol Meas. 2011 Dec;32(12):2003-18

[14]Actibelt "Balance App" available for Android platforms.

https://www.biomedtown.org/biomed town/vphop/rec eption/news/actibelt-201cbalance-app201d-availablefor-android-platforms/ 